\title{
Microsecond resolution timing with Sun workstations
}

\author{
PETER B. DANZIG \\ University of Southern California, Los Angeles, California \\ and \\ STEVE MELVIN \\ University of California, Berkeley, California
}

\begin{abstract}
A high-resolution clock is often indispensable for the accurate measurement of millisecond or microsecond resolution intervals, and it is impossible to measure the probability distribution of short intervals without one. This paper describes a microsecond clock for Sun 3 and 4 workstations that we designed, built, and have made publicly available. It also explains how to measure intervals shorter than a computer's clock resolution, and derives confidence intervals for such measurements.
\end{abstract}

A high-resolution clock is often indispensable for the accurate measurement of millisecond or microsecond resolution intervals, and it is impossible to measure the probability distribution of short intervals without one. This paper describes a microsecond clock that we designed and built for Sun 3 and 4 workstations ${ }^{1}$ and derives confidence intervals for measurements made with lowresolution clocks.

Beginning with its Sun 3 workstations, Sun Microsystems substituted an Intersil, battery backed-up, timeof-day clock chip for the microsecond resolution clock chip present in its earlier models. The new clock interrupts the processor every $10 \mathrm{msec}$. By default, the Sun operating system discards every other interrupt, degrading the clock resolution from 10 to $20 \mathrm{msec}$. The hardware of Sun 3 and 4 workstations supports a data encryption (DES) chip, but the federal government pressured Sun to eliminate the DES chips from their products. For this reason, the DES chip's IC socket is empty in these workstations. Because we had no use for encryption, we built a high-resolution clock on a circuit board that plugs directly into the DES chip's socket. The high-resolution clock is easily installed. One simply inserts our circuit board into the DES chip's socket, inserts from zero to three additional machine-specific support chips into their respective sockets, and compiles the device driver into the operating system. We list the additional support chips in Table 1. We finished the clock in May 1989. In October 1990, more than 70 of our clocks were in use in six countries.

This work has been supported in part by the Defense Advanced Research Projects Agency (DOD), ARPA Order No. 4871, monitored by the Naval Electronic Systems Command under Contract No. N0008984-C-0089. Correspondence should be addressed to Peter B. Danzig, Computer Science Department, University of Southern California, Los Angeles, CA 90089-0782. (E mail: danzig@pollux.usc.edu)
Table 1

Required Support Chips to Activate the DES Socket

\begin{tabular}{ll}
\hline \multicolumn{1}{c}{ Workstation } & \multicolumn{1}{c}{ Support Chips } \\
\hline Sun 3/50, 3/60 & None required \\
Sun 3/75, 3/150, 3/160 & 74ALS245 and PAL22V10 \\
Sun 3/260, 3/280 & 74ALS245, PAL16R4, and PAL16R8 \\
Sun 4/110, 4/150 & PAL22V10 \\
Sun 4/260, 4/280 & PAL22V10 \\
\hline
\end{tabular}

Note-PAL chips require programming.

In the next section, we describe our clock's design. Following the Design section, we derive the number of measurements needed to report average timing measurements as a function of clock resolution and confidence level. In the final section of this paper, we briefly discuss alternative methods to make millisecond resolution measurements on Sun workstations.

\section{MICROSECOND TIMING WITH SUN WORKSTATIONS}

In this section, we describe our clock's design. Because Sun guards its workstations' schematics, we designed our clock to meet the timing requirements and 8-bit interface of the Advanced Micro Devices Am9518 DES chip (also known as the Zilog Z8068). We built the clock around the Am9513a counter chip because its five 16-bit counters can be simultaneously saved with a single instruction yet can be read or written over an 8-bit bus. While other counter chips have multiple 16-bit timer registers, the Am9513a is the only chip that can simultaneously save more than one timer register. Although the timer and DES chips carry similar designations, their pin assignments, interface protocol, and timing needs are quite different.

\section{Design}

The microprocessor interfaces of the 9518 and the 9513 are similar in that they both have a control port and a data 
port, both of which can be read or written. Unfortunately however, the control signals are fundamentally different. The 9518 has two strobe lines, one for the control port and another for the data port, and a read/write signal that determines the direction of the access. The 9513 , on the other hand, has separate strobe lines for read and write and a single control/data signal that determines which port will be accessed. The 9518's read/write signal must meet setup and hold times around the control and data strobes, whereas the 9513's control/data signal must meet setup and hold times around the read and write strobes.

In designing the timer board, we assumed that the signals that Sun workstations generate to access the 9518 only meet the minimum values required by the chip's specifications. We did not have access to Sun processor schematics or timing diagrams, and we wanted the board to work in any model that could take a 9518 . Under these restrictions, it was not possible to map the ports from the 9518 to those of the 9513 in a straightforward way and still meet the setup time on the control/data line. Therefore, we added a simple latch, implemented by a PAL16L8, which switches the control/data line based on accesses to the 9518 data port. A read from the data port sets the control/ data line for a control operation; a write to the data port sets the line for a data operation. Accesses to the control port of the 9518 are then used to access both the control and data ports of the 9513. Note that our scheme requires an additional access whenever the control/data mode is changed. The timer's schematic is shown in Figure 1. The complete circuit used to translate the 9518 signals to 9513 signals is shown in Figure 2.

The timer board's device driver sets the timer chip's fifth timer to divide the 4.0-MHz oscillator frequency by four, concatenates the timer chip's lower four timers, and drives the lowest of these with the output of the fifth timer. The board can return a simple binary count or a 64 -bit
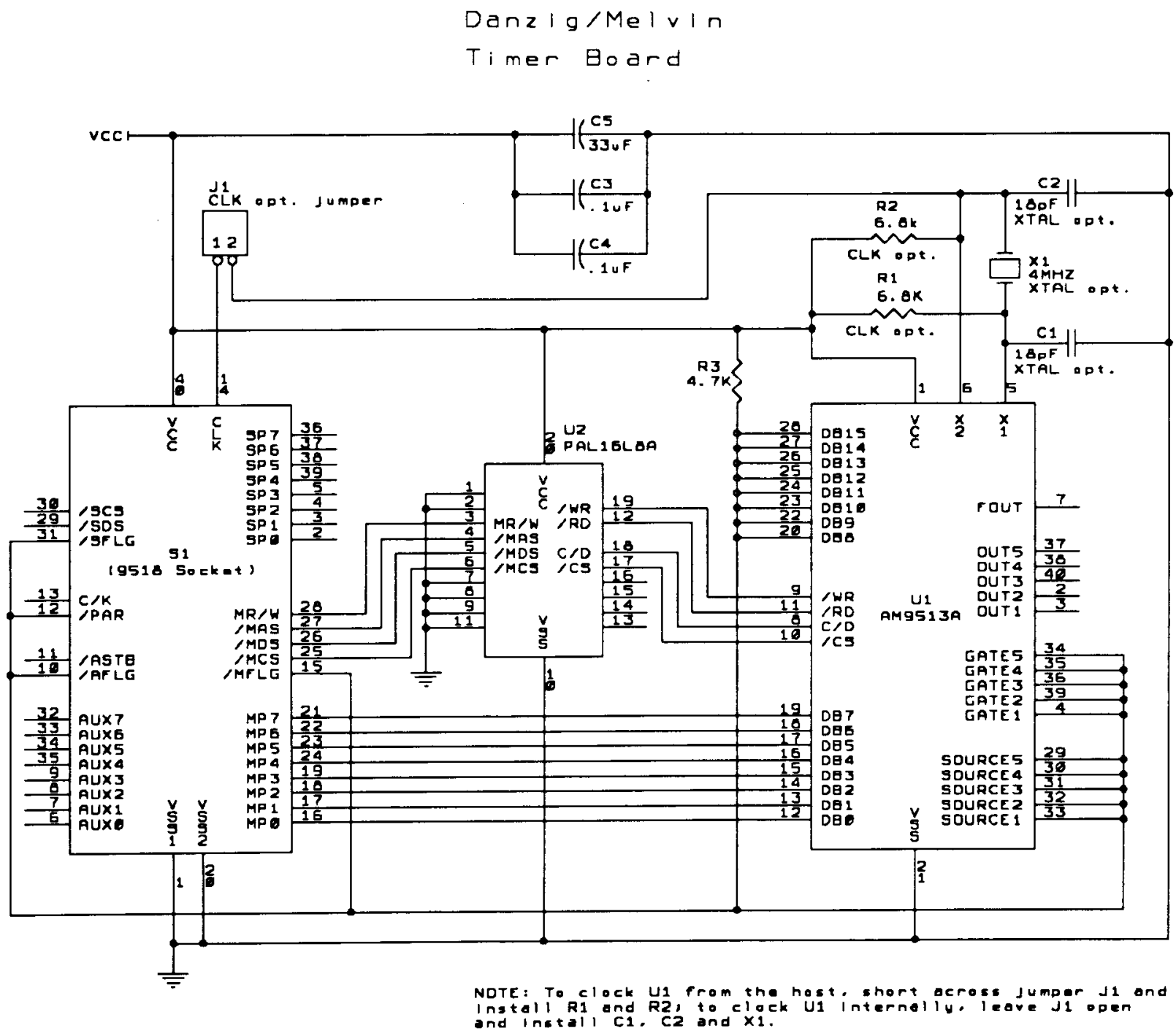

Figure 1. Schematic of the Sun timer board. The chip to the left is actually the empty DES socket on the Sun processor board. The 16L8 PAL debounces and translates the DES chip's control signals into the timer chip's control signals. 


$$
\text { PAL Equivalent Circuit }
$$

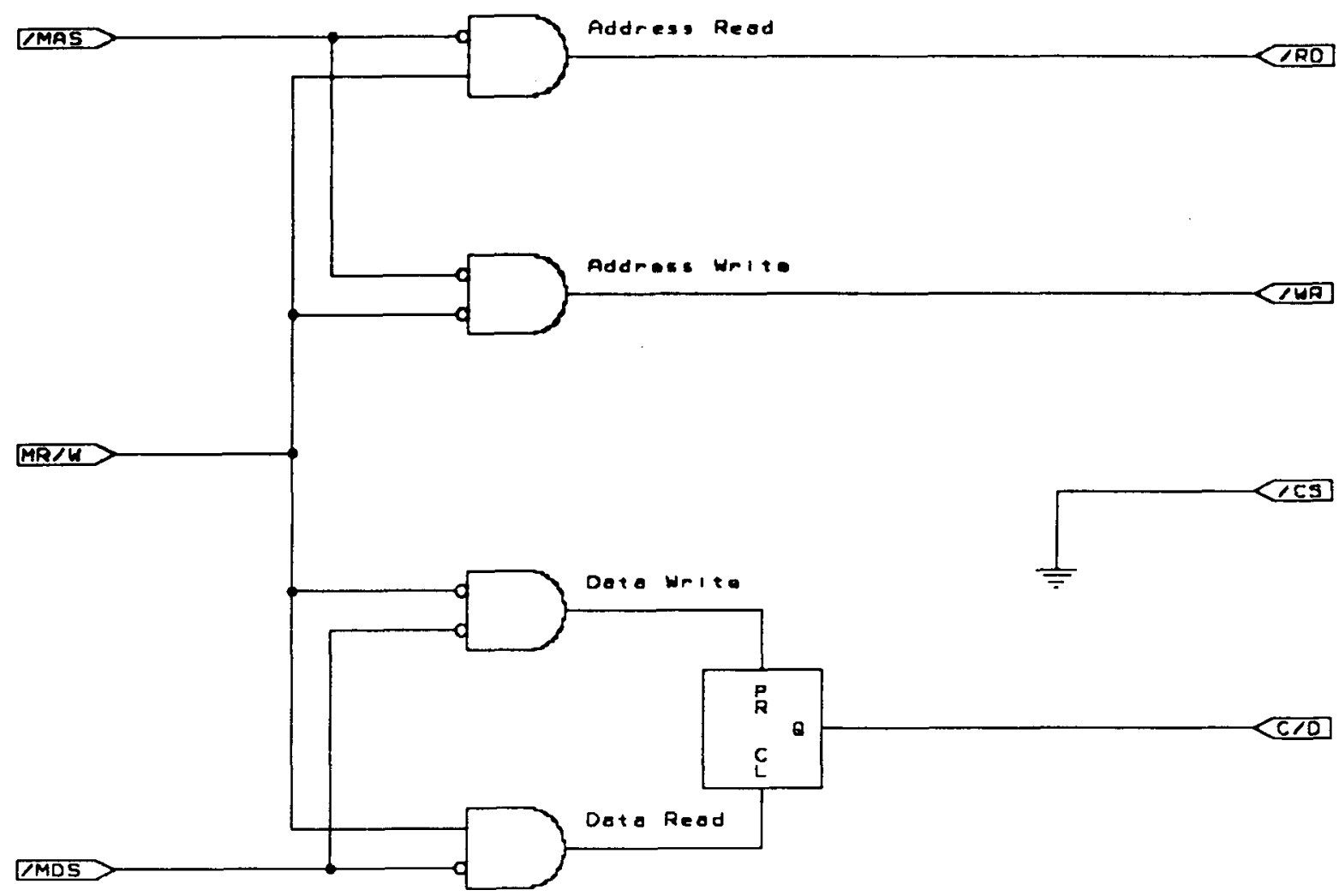

Figure 2. Equivalent circuit for the 16L8 PAL.

UNIX timeval structure. The timeval mode is useful for compatibility with the UNIX system call gettimeofday().

\section{Making Timing Measurements with UNIX}

The clock can be read several different ways. Because it appears as device /dev/tmrO in the file system, users can read or write it as if it were a simple file. This method is slow because it traverses hundreds of lines of UNIX file system code. For this reason, we added a system call to read the clock. Reading the clock through the system call is twice as fast as reading it through the file system. For even lower overhead access to the clock, we modified the device driver to map the clock's registers into the user program's address space. The calls to read the memory mapped timer are actually macros. This option is 10 times faster than the system call, but it suffers a drawback. Since it takes several instructions to read the time and since user programs cannot disable interrupts, user programs may be interrupted while reading the clock. A user program may read a nonsensical value if it is descheduled while accessing the clock, and some other process reads the clock. The other process resets the timer chip's internal pointer, so the original process, when it resumes, continues reading bytes from where it left off, unaware that it is reading the wrong ones. For this reason, unless microsecond accuracy is needed, we recommend accessing the clock through the system call. The clock can be read inside the operating system by making a macro call. This ability is important if the workstation interfaces to other laboratory equipment.

In Table 2, we report the overhead associated with reading 32-bit timestamps and 64-bit timevals from inside the operating system and from user programs. These numbers were measured by reading the timer in a tight loop 10,000 times. We made no attempt to limit interference from other processes. The measurements for the Sun $3 / 260$ were not made by us, but were sent to us by users of the timer board. Note that a 3/50's display steals cycles from main memory, because it does not have a separate frame buffer. Hence, we give two sets of overhead figures for it, one for when the display is blanked and another for when it is active. When the display is active, the machine slows down by more than $20 \%$.

The overhead varies a few microseconds from read to read due to variations in memory access times, memory contention from the display and DMA devices such as the 
Table 2

Measured Overhead (in microseconds) to Read the High-Resolution Clock

\begin{tabular}{lrrrr}
\multicolumn{1}{c}{ Timestamp Type } & $3 / 50$ & $\begin{array}{c}3 / 50 \\
\text { Blanked }\end{array}$ & $3 / 60$ & $3 / 260$ \\
\hline Operating System 32-bit Macro & 24.0 & 19.5 & 16.5 & 11.0 \\
Operating System 64-bit Macro & 38.2 & 30.2 & 27.0 & 18.0 \\
User 32-bit Memory Mapped Macro & 14.0 & 11.3 & 11.1 & 7.0 \\
User 64-bit Memory Mapped Macro & 27.0 & 23.5 & 21.0 & 13.0 \\
User 32-bit System Call & 238.0 & 179.0 & 140.0 & 87.0 \\
User 64-bit System Call & 254.0 & 190.0 & 162.0 & 91.0 \\
\hline
\end{tabular}

Note-We do not report overhead to read a timestamp through the file system because the System Call interface is much preferable.

Ethernet interface, and differing instruction buffer, data cache; and translation lookaside buffer hit rates. Timing errors on the order of milliseconds can occur if a user process is descheduled during an experiment due to interrupts or multiprogramming timeslices. A competing process's time slice can last $100 \mathrm{msec}$; disk and network interrupts can take 2 to $3 \mathrm{msec}$ to process. Fortunately, it is usually possible to eliminate these sources of error by disabling processes that compete with our measurements and by disconnecting the Ethernet connection. (On Sun $3 / 50$ s, the display should be blanked if this does not interfere with the experiment.) In rare cases, you may have to place the measurement code inside the operating system. You can measure keyboard reaction times by generating timestamps inside the operating system's keyboard UARTS's device driver, which is serviced at interrupt priority and is not subject to descheduling. Unfortunately, this requires access to operating system source code.

It is necessary to examine timestamps generated by UNIX workstations to guarantee that your precautions to prevent descheduling are succeeding. We have found that, although you cannot ignore such problems on UNIX systems, it is possible to overcome them. A seldom mentioned trait of the UNIX scheduler is that it gives priority to processes that were blocked and become ready to execute. If your process was blocked for a keystroke, it will almost always execute immediately when the keystroke occurs.

This concludes the hardware and software description of our microsecond clock. The clock hardware and software are being used by about 60 different research groups. The clock's software has been ported to several other operating systems, including the V-System, ATT System-V UNIX, Sprite, and XINU. In the next section, we consider how to measure averages time intervals accurately without a high-resolution clock, as is frequently done to measure the execution time of sections of computer programs.

\section{LOW-RESOLUTION CLOCKS AND CONFIDENCE INTERVALS}

If an experiment is quickly set up and infinitely repeatable, a high-resolution clock may not be necessary. It may be possible to repeat low-resolution measurements hundreds or thousands of times, averaging the results. Here, we derive confidence intervals for measurements taken this way, given that the clock advances every $\Delta$ milliseconds.
One measures short intervals by recording the difference in the clock's values between the beginning and the end of the interval. Since the clock only advances every $\Delta$ milliseconds, it may not advance during an experiment of duration less than $\Delta$ milliseconds. Without loss of generality, assume that we want to measure an interval of duration $\delta \leq \Delta$ milliseconds. (We must assure ourselves that the measurements are not initiated by or otherwise synchronous to the clock ticks. If the experimental process sleeps, or relinquishes the processor, it is quite likely that this technique will not work.) During an interval of duration $\delta$, the clock advances with probability $p=\delta / \Delta$. Define the indicator variable $\sigma_{i}$ to be one if the clock advances during measurement $i$ and zero otherwise. After measuring an interval $n$ times, the clock advances $S_{n}=\sum_{i=1}^{n} \sigma_{i}$ ticks, for which we would estimate the interval's average as $\hat{\delta}=\hat{p} \Delta=S_{n} \Delta / n$.

Let us consider the precision of measurements made in this manner. Notice that the $\sigma_{i}$ S approximate the two possible outcomes of a sequence of Bernoulli trials. We say approximate because the outcomes of subsequent trials may not be totally independent. That is, if we know that the clock advanced during the last measurement, this may increase the probability that the clock does not advance during this measurement. Assuming independence, we want to know how many measurements are required to report these times to two or three significant digits. Since our measurements result from randomized experiments, we can only make probabilistic statements about their precision. Conceivably, but with low probability, we may never observe a single clock tick. Let $\delta=p \Delta$ be the true interval length and $\delta$ be our estimate of it based upon $n$ observations. We would like to report our experimental data as

$$
P(-\epsilon \leq(\hat{\delta}-\delta) \leq \epsilon) \geq \alpha .
$$

That is, with probability $\alpha$ or greater, our measurement is within $\epsilon$ of the true value. We can apply the DeMoivreLaplace limit theorem to find the minimum number of measurements required to make such a statement. The DeMoivre-Laplace theorem (see Feller, 1970) states

$$
\lim _{n \rightarrow \infty} P\left\{z_{1} \sqrt{n p q} \leq S_{n}-n p \leq z_{2} \sqrt{n p q}\right\}=\Phi\left(z_{2}\right)-\Phi\left(z_{1}\right),
$$

where $q=1-p$ and $\Phi(x)$ is the normal distribution function. 
Table 3

Required Number of Measurements to Meausre Intervals of Duration $\delta$ to \pm 1 Unit in the Least Significant Digit, Given a Clock with $\Delta=20$-msec Resolution to a Confidence Level of $\alpha$

\begin{tabular}{cccc}
\hline$\delta$ & $n_{3}$ & $n_{2}$ & $\alpha$ \\
\hline $1 \cdot 10^{1} \mu \mathrm{sec}$ & $8 \cdot 10^{7}$ & $8 \cdot 10^{3}$ & 0.95 \\
$1 \cdot 10^{2} \mu \mathrm{sec}$ & $8 \cdot 10^{6}$ & $8 \cdot 10_{4}$ & 0.95 \\
$1 \cdot 10^{3} \mu \mathrm{sec}$ & $7 \cdot 10^{5}$ & $7 \cdot 10^{3}$ & 0.95 \\
$1 \cdot 10^{4} \mu \mathrm{sec}$ & $4 \cdot 10^{4}$ & $4 \cdot 10^{2}$ & 0.95 \\
\hline
\end{tabular}

We can combine these last two equations using the identity $\Phi(-z)=1-\Phi(z)$ :

$$
\lim _{n \rightarrow \infty} P\left\{\frac{-\epsilon n}{\Delta} \leq S_{n}-n p \leq \frac{\epsilon n}{\Delta}\right\}=2 \Phi\left(z_{\alpha}\right)-1 .
$$

Equating $\epsilon n / \Delta$ with $z_{\alpha} \sqrt{n p q}$ and recalling the definitions $q=1-p$ and $p=\delta / \Delta$, we arrive at an expression for $n$ :

$$
n=\frac{z_{a}^{2} \delta(\Delta-\delta)}{\epsilon^{2}}
$$

for which we must evaluate $z_{\alpha}=\Phi^{-1}[(1+\alpha) / 2]$.

In Table 3, we summarize the number of measurements $n_{2}$ and $n_{3}$ required to report times to two and three decimal places such that the least significant digit is within one unit of the true value with probability $\alpha=0.95$.

\section{CONCLUSIONS}

We note that it is possible to build a clock such as ours in place of nonessential chips in other computers. Alternatively, installations with operating system source code licenses may consider modifying their serial port's device driver to generate a periodic interrupt (see Tzou \& Anderson, 1988). Since most serial interface chips can gener- ate an interrupt whenever a character has been sent, one can set the baud rate so that a character completion interrupt occurs every millisecond or half millisecond. However, this technique not only usurps your serial port, but can impose significant overhead on the system and introduce subtle timing errors.

When it is necessary to measure an interval's distribution rather than average, a high-resolution clock may be essential. The microsecond resolution clock described in this paper will work in all Sun workstations equipped with the DES socket. The clock's device driver has been ported to several operating systems including the V-System, ATT System-V, Sprite, and XINU.

It is possible to make high-resolution measurements without a high-resolution clock if the experiment can be repeated hundreds or thousands of times. Equation $1 \mathrm{ex}$ presses the relationship among interval length, clock resolution, desired confidence level, and number of necessary measurements.

\section{REFERENCES}

FelLeR, W. (1970). An Introduction to probability theory and its applications (Vol. 1. 3rd ed.). New York: Wiley.

Tzou, S.-Y., \& ANDERson, D. P. (1988). Performance evaluation of the DASH message-passing system (Tech. Rep. UCB/CSD 88/452). Berkeley, University of California, Computer Science Division.

\section{NOTE}

1. Contact the authors to obtain the Sun device driver or completed timer boards. Note that the board cannot work in Sun $3 / 80,386 i$, or SPARC stations, but SPARC stations have an internal microsecond timer.

(Manuscript received July 2, 1990; revision accepted for publication October 30, 1990.) 